\title{
Erethism Mercurialis and Reactions to Elemental Mercury
}

\author{
Corey Stone, DO; Jeffrey Angermann, PhD; Jeffrey Sugarman, MD, PhD
}

\section{PRACTICE POINTS}

- Chronic mercury granulomas can present as firm, erythematous to bluish gray plaques.

- Accidental skin contact to elemental mercury may cause urticaria and dermatitis.

- Blood mercury concentrations below $20 \mu \mathrm{g} / \mathrm{L}$ are considered within reference range; once blood and urine concentrations exceed $100 \mu \mathrm{g} / \mathrm{L}$, clinical signs of acute mercury poisoning typically manifest.

- Mercury is toxic to the central and peripheral nervous systems, resulting in erethism mercurialis, a constellation of neuropsychologic signs and symptoms including restlessness, irritability, insomnia, emotional lability, difficulty concentrating, and impaired memory.

Mercury is an underrecognized cause of heavy metal poisoning. Typically, mercury exposure occurs though consumption of methylmercury in seafood or acute inhalation of elemental mercury vapors, with other routes of exposure being uncommon. We describe a case of mercury toxicity resulting from intentional injection of liquid mercury into the right antecubital fossa in a suicide attempt. Mercury poisoning may present with characteristic neuropsychologic signs and symptoms. Increasing anxiety, depression, tremors, irritability, and difficulty concentrating coupled with blood mercury levels higher than $100 \mu \mathrm{g} / \mathrm{L}$ and urine mercury levels of $477 \mu \mathrm{g} / \mathrm{g}$ led to the diagnosis of erethism mercurialis, a constellation of neuropsychologic signs and symptoms including restlessness, irritability, insomnia, emotional lability, difficulty concentrating, and impaired memory. Skin reactions associated with contact to elemental mercury are rare. However, this case presented with a mercury granuloma. Hives and dermatitis have been observed following accidental contact with inorganic mercury compounds.

Cutis. 2021;107:190-192, 198.

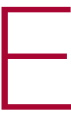

vidence of human exposure to mercury dates as far back as the Egyptians in 1500 BC. ${ }^{1}$ The ancient Chinese believed mercury could prolong life, heal bones, and maintain vitality. ${ }^{2}$ Western medicine has utilized mercury in diuretics, laxatives, antibacterial agents, and antiseptics. ${ }^{3}$ Health effects caused by chronic mercury exposure became increasingly apparent in the 1800s after hat makers who had inhaled mercuric nitrate vapors began to present with a host of neurologic symptoms, which is where the phrase "mad as a hatter" was derived. ${ }^{4,5}$ In 1889, French neurologist Jean-Martin Charcot attributed rapid tremors to mercury poisoning. ${ }^{6}$ By 1940, Kinnier Wilson ${ }^{7}$ further characterized the effects of mercury, describing mercury-induced cognitive impairments. In the 1960s, Japanese researchers correlated elevated urinary mercury levels with an outbreak of Minamata disease, a condition characterized by tremors, sensory loss, ataxia, and visual constrictions. ${ }^{8}$ The World Health Organization considers mercury to be one of the top 10 chemicals of major public health concern. ${ }^{9}$

Mercury release in the environment primarily is a function of human activity, including coal-fired power plants, residential heating, and mining., ${ }^{910}$ Mercury from these sources is commonly found in the sediment of lakes and bays, where it is enzymatically converted to methylmercury by aquatic microorganisms; subsequent food chain biomagnification results in elevated mercury levels in apex predators. Substantial release of mercury into the environment also can be attributed to health care facilities from their use of thermometers containing 0.5 to $3 \mathrm{~g}$ of elemental mercury, ${ }^{11}$ blood pressure monitors, and medical waste incinerators. ${ }^{5}$

Mercury has been reported as the second most common cause of heavy metal poisoning after lead. ${ }^{12}$ Standards from the US Food and Drug Administration dictate that methylmercury levels in fish and wheat products must not exceed $1 \mathrm{ppm} .{ }^{13}$ Most plant and animal food sources contain methylmercury at levels between 0.0001 and $0.01 \mathrm{ppm}$; mercury concentrations are especially high in tuna, averaging $0.4 \mathrm{ppm}$, while larger predatory fish contain levels in excess of $1 \mathrm{ppm} .{ }^{14}$ The use of mercury-containing cosmetic products also presents a substantial exposure risk

Dr. Stone is from the Edward Via College of Osteopathic Medicine, Auburn, South Carolina. Dr. Angermann is from the University of Nevada School of Community Health Sciences, Reno. Dr. Sugarman is from the University of California, San Francisco.

The authors report no conflict of interest.

Correspondence: Jeffrey Sugarman, MD, PhD, 2725 Mendocino Ave, Santa Rosa, CA 95403 (pediderm@yahoo.com). doi:10.12788/cutis.0224 
to consumers. ${ }^{5,10}$ In one study, 3.3\% of skin-lightening creams and soaps purchased within the United States contained concentrations of mercury exceeding 1000 ppm. ${ }^{15}$

We describe a case of mercury toxicity resulting from intentional injection of liquid mercury into the right antecubital fossa in a suicide attempt.

\section{Case Report}

A 31-year-old woman presented to the family practice center for evaluation of a firm stained area on the skin of the right arm. She reported increasing anxiety, depression, tremors, irritability, and difficulty concentrating over the last 6 months. She denied headache and joint or muscle pain. Four years earlier, she had broken apart a thermometer and injected approximately $0.7 \mathrm{~mL}$ of its contents into the right arm in a suicide attempt. She intended to inject the thermometer's contents directly into a vein, but the material instead entered the surrounding tissue. She denied notable pain or itching overlying the injection site. Her medications included aripiprazole and buspirone. She noted that she smoked half a pack of cigarettes per day and had a history of methamphetamine abuse. She was homeless and unemployed. Physical examination revealed an anxious tremulous woman with an erythematous to bluish gray, firm plaque on the right antecubital fossa (Figure 1). There were no notable tremors and no gait disturbance.

Her blood mercury level was greater than $100 \mu \mathrm{g} / \mathrm{L}$ and urine mercury was $477 \mu \mathrm{g} / \mathrm{g}$ (reference ranges, 1-8 $\mu \mathrm{g} / \mathrm{L}$ and $4-5 \mu \mathrm{g} / \mathrm{L}$, respectively). A radiograph of the right elbow area revealed scattered punctate foci of increased density within or overlying the anterolateral elbow soft tissues. She was diagnosed with mercury granuloma causing chronic mercury elevation. She underwent excision of the granuloma (Figure 2) with endovascular surgery via an elliptical incision. The patient was subsequently lost to follow-up.

\section{Comment}

Elemental mercury is a silver liquid at room temperature that spontaneously evaporates to form mercury vapor, an invisible, odorless, toxic gas. Accidental cutaneous exposure typically is safely managed by washing exposed skin

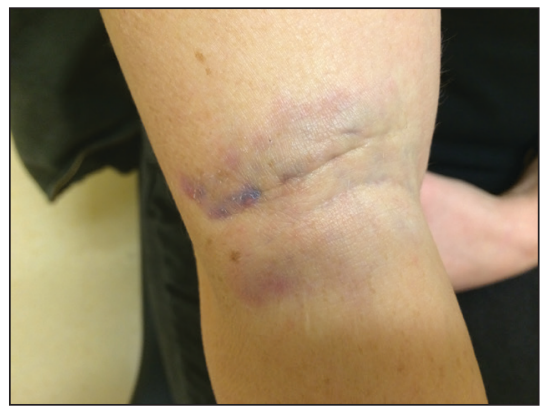

FIGURE 1. Erethism mercurialis. Bluish gray-stained area on the skin of the patient's right antecubital fossa. with soap and water, ${ }^{16}$ though there is a potential risk for systemic absorption, especially when the skin is inflamed. When metallic mercury is subcutaneously injected, it is advised to promptly excise all subcutaneous areas containing mercury, regardless of any symptoms of systemic toxicity. Patients should subsequently be monitored for signs of both central nervous system (CNS) and renal deficits, undergo chelation therapy when systemic effects are apparent, and finally receive psychiatric consultation and treatment when necessary. ${ }^{17}$

Inorganic mercury compounds are formed when elemental mercury combines with sulfur or oxygen and often take the form of mercury salts, which appear as white crystals. ${ }^{16}$ These salts occur naturally in the environment and are used in pesticides, antiseptics, and skin-lightening creams and soaps. ${ }^{18}$

Methylmercury is a highly toxic, organic compound that is capable of crossing the placental and blood-brain barriers. It is the most common organic mercury compound found in the environment. ${ }^{16}$ Most humans have trace amounts of methylmercury in their bodies, typically as a result of consuming seafood. ${ }^{5}$

Exposure to mercury most commonly occurs through chronic consumption of methylmercury in seafood or acute inhalation of elemental mercury vapors. ${ }^{9}$ Iatrogenic cases of mercury exposure via injection also have been reported in the literature, including a case resulting in acute poisoning due to peritoneal lavage with mercury bichloride. ${ }^{19}$ Acute mercury-induced pulmonary damage typically resolves completely. However, there have been reported cases of exposure progressing to interstitial emphysema, pneumatocele, pneumothorax, pneumomediastinum, interstitial fibrosis, and chronic respiratory insufficiency, with examples of fatal acute respiratory distress syndrome being reported. 5,16,20 Although individuals who inhale mercury vapors initially may be unaware of exposure due to little upper airway irritation, symptoms following an initial acute exposure may include ptyalism, a metallic taste, dysphagia, enteritis, diarrhea, nausea, renal damage, and CNS effects. ${ }^{16}$ Additionally, exposure may lead to confusion with signs and symptoms of metal fume fever, including

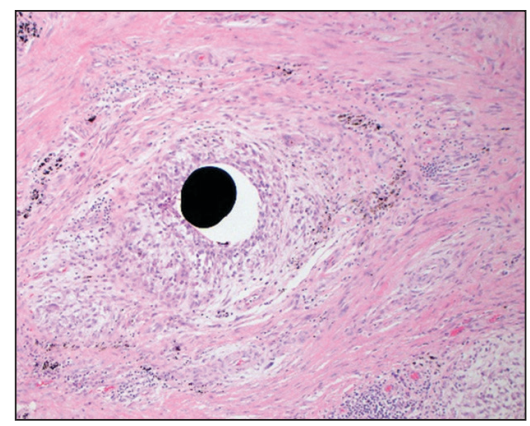

FIGURE 2. Histopathology showed a mercury granuloma (H\&E, original magnification $\times 20$ ). 
shortness of breath, pleuritic chest pain, stomatitis, lethargy, and vomiting. ${ }^{20}$

Chronic exposure to mercury vapor can result in accumulation of mercury in the body, leading to neuropsychiatric, dermatologic, oropharyngeal, and renal manifestations. Sore throat, fever, headache, fatigue, dyspnea, chest pain, and pneumonitis are common. ${ }^{16}$ Typically, low-level exposure to elemental mercury does not lead to long-lasting health effects. However, individuals exposed to high-level elemental mercury vapors may require hospitalization. Treatment of acute mercury poisoning consists of removing the source of exposure, followed by cardiopulmonary support. ${ }^{16}$

Specific assays for mercury levels in blood and urine are useful to assess the level of exposure and risk to the patient. Blood mercury concentrations of $20 \mu \mathrm{g} / \mathrm{L}$ or below are considered within reference range; however, once blood and urine concentrations of mercury exceed $100 \mu \mathrm{g} / \mathrm{L}$, clinical signs of acute mercury poisoning typically manifest. ${ }^{21}$ Chest radiographs can reveal pulmonary damage, while complete blood cell count, metabolic panel, and urinalysis can assess damage to other organs. Neuropsychiatric testing and nerve conduction studies may provide objective evidence of CNS toxicity. Assays for $\mathrm{N}$-acetyl- $\beta$-D-glucosaminidase can provide an indication of early renal tubular dysfunction. ${ }^{16}$

Elemental mercury is not absorbed from the gastrointestinal tract, posing minimal risk for acute toxicity from ingestion. Generally, less than $10 \%$ of ingested inorganic mercury is absorbed from the gut, while elemental mercury is nonabsorbable. ${ }^{10}$ If an individual ingests a large amount of mercury, it may persist in the gastrointestinal tract for an extended period. Mercury is radiopaque, and abdominal radiographs should be obtained in all cases of ingestion. ${ }^{16}$

Mercury is toxic to the CNS and peripheral nervous system, resulting in erethism mercurialis, a constellation of neuropsychologic signs and symptoms including restlessness, irritability, insomnia, emotional lability, difficulty concentrating, and impaired memory. In severe cases, delirium and psychosis may develop. Other CNS effects include tremors, paresthesia, dysarthria, neuromuscular changes, headaches, polyneuropathy, and cerebellar ataxia, as well as ophthalmologic and audiologic impairment. ${ }^{5,16}$

Upon inhalation exposure, patients with respiratory concerns should be given oxygen. Bronchospasms are treated with bronchodilators; however, if multiple chemical exposures are suspected, bronchial-sensitizing agents may pose additional risks. Corticosteroids and antibiotics have been recommended for treatment of chemical pneumonitis, but their efficacy has not been substantiated. ${ }^{16}$

Skin reactions associated with skin contact to elemental mercury are rare. However, hives and dermatitis have been observed following accidental contact with inorganic mercury compounds. ${ }^{5}$ Manifestation in children chronically exposed to mercury includes a nonallergic hypersensitivity (acrodynia), ${ }^{5,17}$ which is characterized by pain and dusky pink discoloration in the hands and feet, most often seen in children chronically exposed to mercury absorbed from vapor inhalation or cutaneous exposure. ${ }^{16}$

Renal conditions associated with acute inhalation of elemental mercury vapor include proteinuria, nephrotic syndrome, temporary tubular dysfunction, acute tubular necrosis, and oliguric renal failure. ${ }^{16}$ Chronic exposure to inorganic mercury compounds also has been reported to cause renal damage. ${ }^{5}$ Chelation therapy should be performed for any symptomatic patient with a clear history of acute elemental mercury exposure. ${ }^{16}$ The most frequently used chelation agent in cases of acute inorganic mercury exposures is dimercaprol. In rare cases of mercury intoxication, hemodialysis is required in the treatment of renal failure and to expedite removal of dimercaprol-mercury complexes. ${ }^{16}$

Cardiovascular symptoms associated with acute inhalation of high levels of elemental mercury include tachycardia and hypertension. ${ }^{16}$ Increases in blood pressure, palpitations, and heart rate also have been observed in instances of acute elemental mercury exposure. Studies show that exposure to mercury increases both the risk for acute myocardial infarction as well as death from coronary heart and cardiovascular diseases. ${ }^{5}$

\section{Conclusion}

Mercury poisoning presents with varied neuropsychologic signs and symptoms. Our case provides insight into a unique route of exposure for mercury toxicity. In addition to the unusual presentation of a mercury granuloma, our case illustrates how surgical techniques can aid in removal of cutaneous reservoirs in the setting of percutaneous exposure.

\section{REFERENCES}

1. History of mercury. Government of Canada website. Modified April 26, 2010. Accessed March 11, 2021. https://www.canada.ca /en/environment-climate-change/services/pollutants/mercury -environment/about/history.html

2. Dartmouth Toxic Metals Superfund Research Program website. Accessed March 11, 2021. https://sites.dartmouth.edu/toxmetal/

3. Norn S, Permin H, Kruse E, et al. Mercury-a major agent in the history of medicine and alchemy [in Danish]. Dan Medicinhist Arbog. 2008;36:21-40.

4. Waldron HA. Did the Mad Hatter have mercury poisoning? Br Med J (Clin Res Ed). 1983;287:1961.

5. Poulin J, Gibb H. Mercury: assessing the environmental burden of disease at national and local levels. WHO Environmental Burden of Disease Series No. 16. World Health Organization; 2008.

6. Charcot JM. Clinical lectures of the diseases of the nervous system. In: Kinnier Wilson SA. The Landmark Library of Neurology and Neurosurgery. Gryphon Editions; 1994:186.

7. Kinnier Wilson SA. Neurology. In: Kinnier Wilson SA. The Landmark Library of Neurology and Neurosurgery. Gryphon Editions; 1994:739-740.

8. Harada M. Minamata disease: methylmercury poisoning in Japan caused by environmental pollution. Crit Rev Toxicol. 1995;25:1-24.

9. Mercury and health. World Health Organization website. Updated March 31, 2017. Accessed March 12, 2021. http://www.who int/mediacentre/factsheets/fs361/en/

10. Olson DA. Mercury toxicity. Updated November 5, 2018. Accessed March 12, 2021.http://emedicine.medscape.com/article/1175560-overview

CONTINUED ON PAGE 198 


\section{ERETHISM MERCURIALIS}

CONTINUED FROM PAGE 192

11. Mercury thermometers. Environmental Protection Agency website. Updated June 26, 2018. https://www.epa.gov/mercury /mercury-thermometers

12. Jao-Tan C, Pope E. Cutaneous poisoning syndromes in children: a review. Curr Opin Pediatr. 2006;18:410-416.

13. US Department of Health and Human Services: Public Health Service Agency for Toxic Substances and Disease Registry. Toxicological profile for mercury: regulations and advisories. Published March 1999. Accessed March 23, 2021. https://www.atsdr.cdc.gov/toxprofiles/tp46.pdf

14. US Food and Drug Administration. Mercury levels in commercial fish and shellfish (1990-2012). Updated October 25, 2017. Accessed March 16, 2021. https://www.fda.gov/food/metals-and-your-food /mercury-levels-commercial-fish-and-shellfish-1990-2012

15. Hamann CR, Boonchai W, Wen L, et al. Spectrometric analysis of mercury content in 549 skin-lightening products: is mercury toxicity a hidden global health hazard? J Am Acad Dermatol. 2014;70:281-287.e3.
16. Mercury. Managing Hazardous Materials Incidents. Agency for Toxic Substances and Disease Registry website. Accessed March 16, 2021. https://www.atsdr.cdc.gov/MHMI/mmg46.pdf

17. Krohn IT, Solof A, Mobini J, et al. Subcutaneous injection of metallic mercury. JAMA. 1980;243:548-549.

18. Lai O, Parsi KK, Wu D, et al. Mercury toxicity presenting acrodynia and a papulovesicular eruption in a 5-year-old girl. Dermatol Online J. 2016;16;22:13030/qt6444r7nc.

19. Dolianiti M, Tasiopoulou K, Kalostou A, et al. Mercury bichloride iatrogenic poisoning: a case report. J Clin Toxicol. 2016;6:2. doi:10.4172/2161-0495.1000290

20. Broussard LA, Hammett-Stabler CA, Winecker RE, et al. The toxicology of mercury. Lab Med. 2002;33:614-625. doi:10.1309/5HY1V3NE-2LFL-P9MT

21. Byeong-Jin Y, Byoung-Gwon K, Man-Joong J, et al. Evaluation of mercury exposure levels, clinical diagnosis and treatment for mercury intoxication. Ann Occup Environ Med. 2016;28:5. 